\title{
Alternative Contrast Mechanism in Optical Coherence Tomography: Temporal Speckle Synchronization Effects
}

DOI: $10.17691 / \mathrm{stm} 2018.10 .1 .05$

Received November 12, 2017

V. Demidov, PhD Student, Department of Medical Biophysics";

O. Demidova, Art to Science University Transfer Program Student, Department of Arts and Science 2 ;

A. Shabunin, PhD, DSc, Professor, Department of Radiophysics and Nonlinear Dynamics;

I.A. Vitkin, PhD, Professor, Departments of Medical Biophysics ${ }^{1}$ and Radiation Oncology ${ }^{4}$;

Senior Scientist and Clinical Physicist, Princess Margaret Cancer Center ${ }^{5}$

1 University of Toronto, 101 College St., Toronto, ON M5G 1L7, Canada;

${ }^{2}$ Seneca College, 1750 Finch Avenue East, Toronto, ON M2J 2X5, Canada;

${ }^{3}$ Saratov State University, 83 Astrakhanskaya St., Saratov, 410012, Russia;

${ }^{4}$ University of Toronto, 150 College St., Toronto, ON M5S 3E2, Canada;

${ }^{5}$ University Health Network, 610 University Avenue, Toronto, ON M5G 2M9, Canada

We propose an alternative optical coherence tomography (OCT) contrast mechanism based on analysis of speckle temporal synchronization using B-mode OCT structural images. We show that the changes in synchronized speckle intensities with time may be used to distinguish between different tissue types, thus providing a novel and potentially useful contrast for OCT imaging. The developed methodology is tested in scattering flow phantoms, and in vivo on cervical cancer tumour grown within a mouse dorsal skin window chamber model. Derived speckle synchronization metric is compared with autocorrelation function analysis to demonstrate its different nature. The phantom and pre-clinical in vivo results suggest that the proposed synchronization approach is sensitive to tissue type/pathology, potentially enabling tumour quantitative evaluation and its delineation from surrounding normal tissues.

Key words: medical imaging; optical coherence tomography; speckle; synchronization; image contrast; image processing; tissue characterization; temporal decorrelation.

How to cite: Demidov V., Demidova O., Shabunin A., Vitkin I.A. Alternative contrast mechanism in optical coherence tomography: temporal speckle synchronization effects. Sovremennye tehnologii v medicine 2018; 10(1): 39, https://doi.org/10.17691/stm2018. 10.1 .05

\section{Introduction}

Optical coherence tomography (OCT) is an emerging non-invasive imaging modality for visualizing subsurface tissue microstructure in vivo at resolutions approaching histology and blood flow details at the microcirculation level [1]. The obtained OCT images exhibit grainy patterns called speckles [2] produced by interference of coherent waves scattered by object features that are smaller than the OCT spatial resolution. While adding to noise, speckle characteristics are also known to contain useful information related to tissue type [3], cellularity [4], response to therapy [5] and other quantities of interest that are not directly visible nor spatially resolved in structural B-mode OCT images. Potential applications of speckle patterns for quantitative analysis and interpretation of OCT images is currently a subject of growing interest among research community [6-10]. Newly developed and well-established methods have been used to analyse OCT speckle patterns. Spatial statistics [3, 11] and fractal dimension analysis [12, 13] show their potential relevance to cellular/subcellular microstructure of tissue, while temporal statistics quantify changes in underlying tissue viscosity thus enabling sensitive delineation of tissue microvascular network [14-16]. In this study, we consider another novel alternative to quantify the changes in speckle patterns and propose an OCT contrast mechanism based on the speckle temporal statistics.

OCT speckle pattern changes with time depending on the nature of the processes occurring within the living tissue, e.g. intracellular motion or blood flow within the vascular network. The rate of intracellular motion differs for various types of tissue depending on the metabolic state of cells, their viability, ion transport, motion of organelles and other activities [17]. Cellular packing density varying from one type of tissue to

Corresponding author: Valentin Demidov, e-mail: val.demidov@mail.utoronto.ca 
another also plays a significant role in OCT imaging by inducing dynamical changes in speckle patterns [4]. We hypothesize that OCT speckles originating from the areas of the same tissue type/condition (e.g., healthy dermal cellular structures) will behave synchronously in time with certain degree of synchronization. Analogously, degrees of synchronization of speckle patterns from areas of other tissue types/conditions (e.g., blood vessel or melanoma tumour cells within the dermal layer) will be different, thus providing an alternative contrast mechanism derived from conventional OCT images.

The concept of synchronization, an interesting and fundamental property of dynamic systems, was first described by Christiaan Huygens who noticed "an odd kind of sympathy" of coupled clocks and reported his observations to the Royal Society in the XVII century [18]. The classical concept of synchronization considers adjustment of rhythms of oscillating objects due to their coupling strength in terms of amplitude, frequency, and phase [19]. The interactive behaviour of complex systems that is often called "chaotic synchronization" [20] refers to a number of different phenomena [21] such as a transition to identical oscillations in coupled subsystems (complete synchronization of chaos), instantaneous phase locking (phase synchronization), basic frequency locking (frequency synchronization), deterministic relationship between the dynamics of oscillators (generalized synchronization), and others. For each of these phenomena, one can separate cases of full and partial synchronization.

Synchronization theory has been widely applied to the analysis of various biological signals. Newly developed methods for detection/quantification of synchronized behaviour, added to time series analysis for estimation of amplitudes, phases, and frequencies from biomedical data [e.g., 22], provide new insights into the complex dynamics of living systems. Detecting resonant oscillations in arterial blood flow at several characteristic frequencies [23], diagnosing kidney malfunctionality by estimating synchronization in renal cortex dynamics [24], recognizing patterns of rhythmic and chaotic synchronized neural activity [25], synching organ response to periodic and chaotic external excitation (e.g., pacemakers [26] and implantable defibrillators [27]) - these are a few examples of applying synchronization theory research to clinical practice. Motivated by the novelty and successes of this approach, here we apply one of the synchronization theory methods [28, 29] (initially used to identify chaotic signals [30] and to study the electrical activity of human brain [31]) to OCT. The developed synchronization metric is tested on tissue mimicking phantom with controlled biophysical/optical properties. The potential of this approach to differentiate tissue types and provide additional contrast for in vivo tissue OCT imaging is demonstrated with imaging the cervical cancer tumour grown within a mouse dorsal skin window chamber model.

In order to analyse temporal and spatial speckle patterns in complex dynamical systems such as living tissue, we first quantify their interconnection information content in the symbolic way through the mutual information (MI) metric. Consider intensities $x_{1}$ and $x_{2}$ of two adjacent speckle pixels at the same depth in an OCT image (Figure 1 (a)) as stochastic signals evolving in time (Figure 1 (b)). Mutual information $\mathrm{Ml}_{12}$ between them is defined as:

$$
\mathrm{MI}_{12}=\sum_{k, m=1}^{N} \log \left[p_{12}\left(X_{k}, X_{m}\right)\right] \log \frac{p_{12}\left(X_{k}, X_{m}\right)}{p_{1}\left(X_{k}\right) p_{2}\left(X_{m}\right)},
$$

where $p_{1}\left(X_{k}\right)$ is a probability that variable $x_{1}$ takes the value $X_{k}$, while $p_{12}\left(X_{k}, X_{m}\right)$ is a joint probability of fulfillment of simultaneous equalities $X_{1}=X_{k}$ and $x_{2}=X_{m}$, and $N$ is the number of quantization levels.

MI may also be calculated through Shannon [32] and conditional [33] entropies:

$$
\mathrm{MI}_{12}=H\left(x_{2}\right)-H\left(x_{2} \mid x_{1}\right) \text {, }
$$

where $H\left(x_{2}\right)$ is Shannon entropy of $x_{2}$ :

$$
H\left(x_{2}\right)=-\sum_{m=1}^{N} p_{2}\left(X_{m}\right) \log \left[p_{2}\left(X_{m}\right)\right]
$$

and $H\left(x_{2} \mid x_{1}\right)$ is a conditional entropy, calculated from $x_{2}$ signal with the value of $x_{1}$ is defined:

$$
H\left(x_{2} \mid x_{1}\right)=-\sum_{k=1}^{N} p_{1}\left(X_{k}\right) \sum_{m=1}^{N} p_{2}\left(X_{m} \mid X_{k}\right) \log \left[p_{2}\left(X_{m} \mid X_{k}\right)\right] \text {. }
$$

Here $p_{2}\left(X_{m} \mid X_{k}\right)$ is a probability that $x_{2}$ takes value $X_{m}$ if $x_{1}=X_{k}$. From distribution of states of $x_{1}$ and $x_{2}$, conditional and Shannon probabilities are calculated as schematically shown in Figure 1 (c) and (d), and then $\mathrm{Ml}_{12}$ is calculated according to (2).

$\mathrm{MI}_{12}$ characterises the degree of interdependence between the stochastic processes of speckle pattern temporal changes. As implied by Eq. (2), it varies from zero for independent changes, to $H\left(x_{2}\right)$ for deterministically dependent ones. Normalizing $\mathrm{Ml}$ to its maximum value yields the index of synchronization $\mu$ between the speckle pixel intensities:

$$
\mu=\frac{H\left(x_{2}\right)-H\left(x_{2} \mid x_{1}\right)}{H\left(x_{2}\right)} \text {. }
$$

When OCT speckle pixels $x_{1}$ and $x_{2}$ intensities are strongly inter-dependent, their level of synchronization $\mu$ is close to unity. In the case when $x_{1}$ and $x_{2}$ are completely independent, their time series are non-synchronized (zero degree of synchronization) and $\mu$ is equal to zero. In intermediate cases when $x_{1}$ and $x_{2}$ demonstrate partial synchronization, the degree of synchronization assumes values between 0 and 1 .

In the context of in vivo tissue imaging, (5) will likely approach unity in regions with relatively slow processes (e.g., skin). On the other hand, in dynamic regions areas where pixel intensities change rapidly in a chaotic unpredictable manner, $\mu$ is expected to be $\sim 0$ with certain degree of error depending on the number of analysed data points. Between these two extremes, index of 

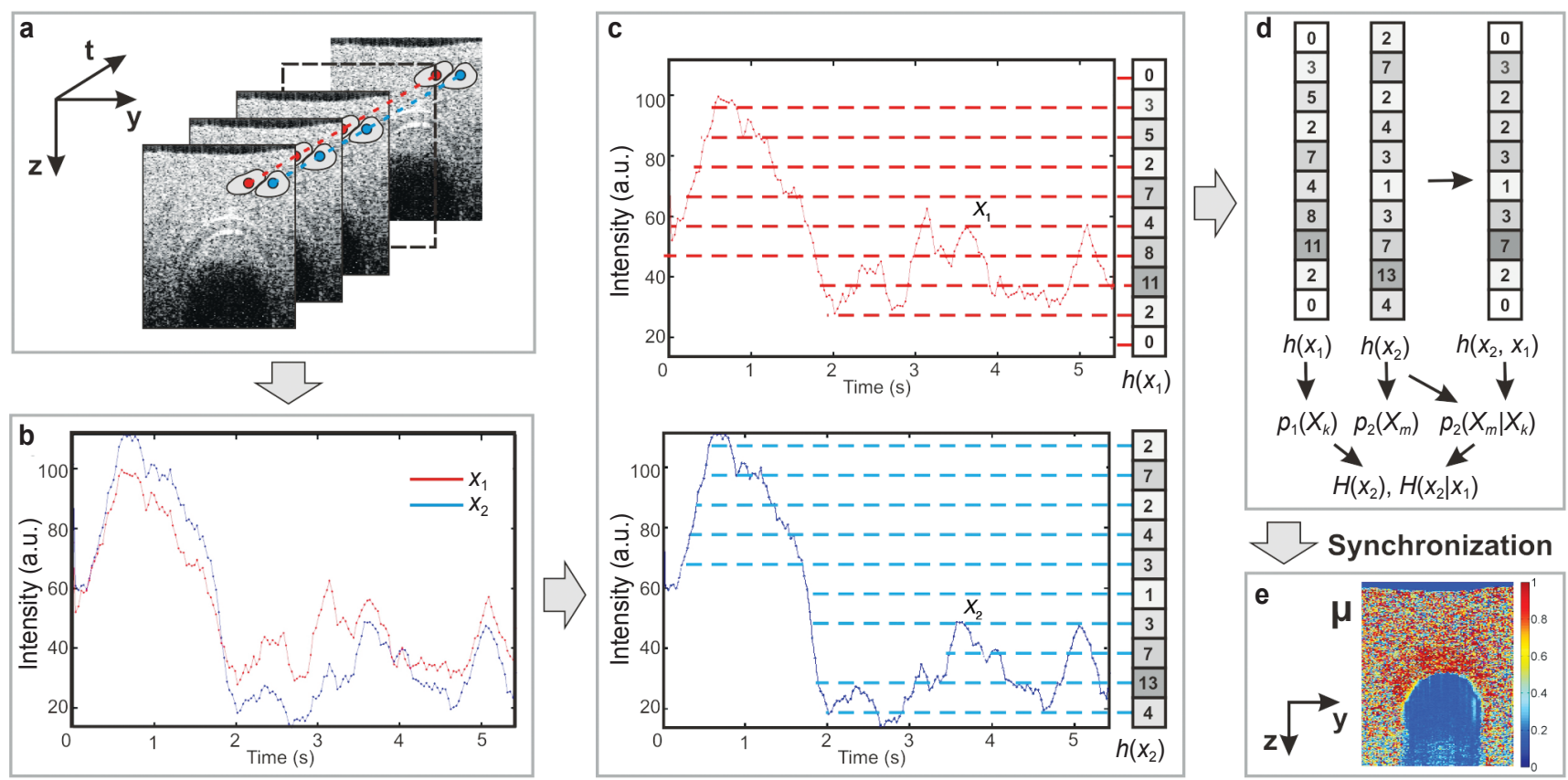

Figure 1. Schematic of the synchronization algorithm

(a) Several consecutive OCT images of agar phantom obtained from the same location, indicating two pixels of neighboring speckles (labeled red and blue dots) chosen for analysis. (b) Time series of $x_{1}$ and $x_{2}$ pixel intensities. (c) Histograms of $h\left(x_{1}\right)$ and $h\left(x_{2}\right)$ intensity distributions; note that the count in each square is equal to number of horizontal signal crossing at that intensity, with corresponding change in square shading level for better visualization (d). Calculation of conditional, non-conditional probabilities and entropies. $p_{2}\left(X_{m} \mid X_{k}\right)$ is a probability that variable $x_{2}$ takes the value $X_{m}$ if $x_{1}=X_{k} \cdot p_{1}\left(X_{k}\right)$ is a probability that variable $x_{1}$ takes the value $X_{k}$ and $p_{2}\left(X_{m}\right)$ is a probability that variable $x_{2}$ takes the value $X_{m}$. $H\left(x_{2}\right)$ is Shannon entropy of $x_{2}, H\left(x_{2} \mid x_{1}\right)$, is a conditional entropy calculated from $x_{2}$ signal if the value of $x_{1}$ is defined. (e) Resulting parametric image, with $\mu$ values from 0 (no synchronization) to 1 (full synchronization)

synchronization can provide additional information about specific tissue dynamics depending on its mutual image speckle behaviour. Note again that $\mu$ differs from the "conventional" single-pixel temporal decorrelation (as quantified by the auto-correlation function analysis), in that it reports on mutual temporal behaviour of two neighbouring pixels; as such, its values may be high or low somewhat independent of the single-pixel temporal dynamics.

\section{Materials and Methods}

Phantom preparation. First experimental phantom consisted of a transparent $600 \mu \mathrm{m}$ inner diameter Teflon microtube immersed in water and filled with a $1 \%$ water suspension of $0.96 \mu \mathrm{m}$ polystyrene microspheres (Bang Laboratories Inc., USA) and driven by a syringe pump (Model NE-4002x; New Era Pump Systems Inc., USA) at a flow rate of $0.86 \mathrm{~mm} / \mathrm{s}$. This phantom system was used to test the methodology under the best controlled conditions and parameters.

The second phantom was constructed to mimic biological tissue with a blood vessel in it. It consisted of the same diameter Teflon microtube imbedded in nearsolid scattering medium (mixture of $1 \%$ agarose gel and
$1 \%$ Intralipid) and is filled with stationary or flowing blood collected from mice. After preparation, phantom was cooled down for two hours at $4^{\circ} \mathrm{C}$ before OCT imaging. This time period ensured that agar + Intralipid scattering medium was not completely solidified, mimicking tissue biomechanical properties.

Mouse tumour model. For in vivo measurements, cross-sectional OCT images were taken from healthy skin and adjacent cervical cancer tumor grown within a dorsal skin window chamber in NRG (NOD-Rag1 ${ }^{\text {null }}$ IL2ry ${ }^{\text {null }}$ ) mice. NRG mice is an immune-compromised hairy strain [34] with eliminated T-cells and radio/chemotherapy resistance, routinely used for the establishment of humanized tumour models for in vivo assessment of anticancer therapies. Human ME-180 cervical cancer cells were cultured in RPMI 1640 medium supplemented with $2 \mathrm{mM}$ L-glutamine, $10 \%$ fetal bovine serum, and $1 \%$ penicillin-streptomycin (Gibco BRL, USA) at $5 \% \mathrm{CO}_{2}$ and $37^{\circ} \mathrm{C}$. ME-180 tumors were grown by injection of $2.5 \cdot 10^{5}$ cells prepared in $10 \mu$ of $1: 1$ PBS:Matrigel (BD Biosciences, Canada) solution into the dorsal skin of seven-week-old NRG mice (Jackson Labs, USA) using a $30 G$ needle. Titanium dorsal skin window chamber surgery was performed 2-3 weeks post injection after the tumour reached $\sim 5 \mathrm{~mm}$ diameter. OCT imaging 
was performed after a recovery period of five days post window chamber installation. Mice were anesthetized with a mixture of $80 \mathrm{mg} / \mathrm{kg}$ of ketamine and $5 \mathrm{mg} / \mathrm{kg}$ of xylazine and placed on a mouse restrainer [35] with built-in $37^{\circ} \mathrm{C}$ heating element to prevent motion artifacts and maintain physiological temperature during imaging procedures as shown in Figure 2. All animal procedures were performed in accordance with the Animal Use Protocol approved by the University Health Network Institutional Animal Care and Use Committee in Toronto, Canada.

OCT instrumentation. Imaging was performed using a fiber-optic swept-source OCT system based on Mach-Zehnder interferometer similar to that described previously [14] and schematically shown in Figure 3. Briefly, a $36 \mathrm{kHz}$ short cavity laser source with a polygonbased tunable filter and wave length centered at $1310 \mathrm{~nm}$

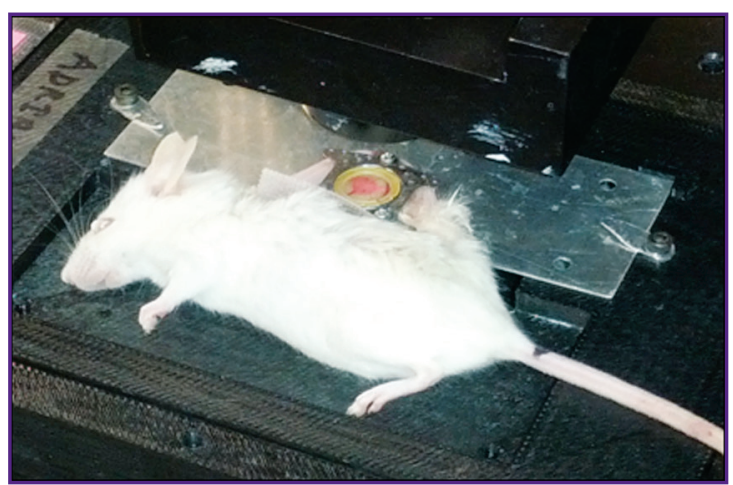

Figure 2. OCT imaging of NRG mouse with cervical cancer tumour grown within a dorsal skin window chamber

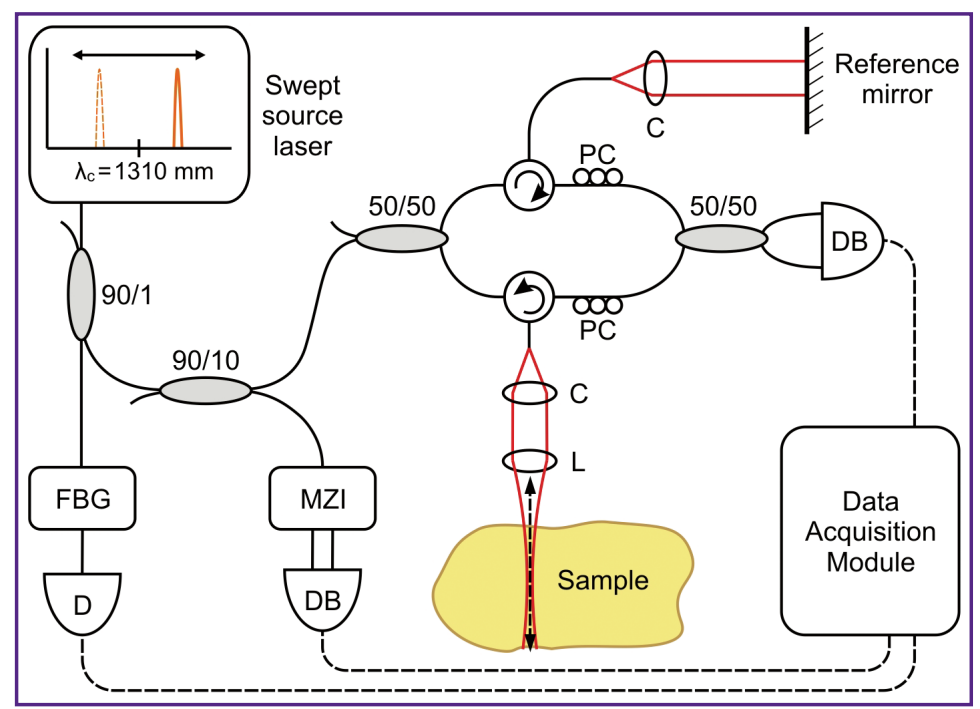

Figure 3. Schematic of the swept-source fiber-optic OCT system based on Mach-Zehnder interferometer

Black lines represent fiber-optics, red lines represent free-space optical paths. PC: polarization controller; D: photo-detector; DB: dual balanced photo-detector; C: collimating lens, L: focusing lens; FBG: fiber Bragg grating; MZI: Mach-Zehnder recalibration interferometer had a sweeping range of $110 \mathrm{~nm}$. The axial resolution (in air) of the system was $\sim 8 \mu \mathrm{m}$ and average output power of $40 \mathrm{~mW}$. Data was sampled (ATS9350; AlazarTech, Canada) with the external clock signal derived at equidistant wavenumber intervals within the sweeping range. Each sweep was triggered by the signal of a fiber Bragg grating centered at $1255 \mathrm{~nm}$ and trigger signal detected with a $125 \mathrm{MHz}$ photodetector. An OCT signal recalibration was performed with a standard MachZehnder recalibration interferometer to generate linear k-space data from the raw data sampled with a $125 \mathrm{MS} / \mathrm{s}$ data acquisition card. Samples were illuminated through a collimated $(C)$ single mode fibre with a lens $(L)$ working distance of $40 \mathrm{~mm}$. The total optical power incident on the sample was $4.6 \mathrm{~mW}$.

The reference arm mirror was illuminated through a fiber collimator. Two fiber circulators were used in both the reference arm and sample arm to redirect the backreflected light to a 50/50 fibre coupler. In order to match the polarization state of the two arms, two polarization controllers were used for adjustment. A $150 \mathrm{MHz}$ (PDB450C; Thorlabs, USA) dual balanced photo-detector was used to detect the output signal from interferometer. Prior to Fourier transformation, the data set was zero padded to 1024 points.

OCT speckle synchronization and relationships to decorrelation times. Post-processing of OCT images was performed using Matlab software (MathWorks, USA). Speckle temporal synchronization was evaluated via the algorithm described in Figure 1 and Eq. (5). A region of interest (ROI) was chosen in each OCT image consisting on tens of pixels that form image speckles as schematically shown in the left square panel of Figure 4. Each square panel in the figure represents the same ROI, different panels showing the schematic selection of the two speckles $x_{1}$ and $x_{2}$ selected for the synchronization analysis. Index of synchronization $\mu$ was estimated for all pairs of ROI speckle pixels (red and blue dots represent a pixel pair in each panel of Figure 4) and then averaged to get a single number for this ROI. A gap of one pixel within each pixel pair insured that analysed pixel intensities were not detected within the same resolution element of the system.

Autocorrelation function (ACF) analysis was also performed to determine speckle decorrelation times, a benchmark approach for characterizing single-pixel sample dynamics, to be compared with the pair-of-pixels synchronization results. Autocorrelation was considered as the correlation between intensity values $I_{i}$ of the same pixel at times $t_{i}$ and $t_{i+\tau}$ with increasing time delay $\tau$ :

$$
\mathrm{ACF}=\frac{\sum_{i=1}^{N-\tau}\left(I_{i}-\bar{l}\right)\left(I_{i+\tau}-\bar{l}\right)}{\sum_{i=1}^{N}\left(I_{i}-\bar{l}\right)^{2}} .
$$




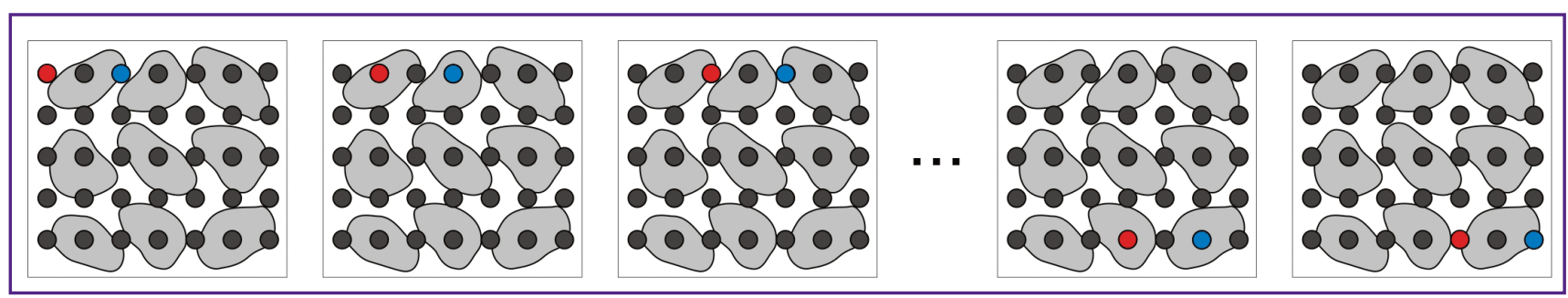

Figure 4. Pixel pairs choice from ROI of OCT structural image for analysis of their temporal synchronization

$\mu$ was estimated for all pairs of ROI speckle pixels (red and blue dots represent a pixel pair in each panel) and then averaged to get a single number for this ROI. A gap of one pixel in between insured that analysed pixel intensities were not detected within the same beam waist. Grey near-oval shapes represent speckle pattern in OCT structural images
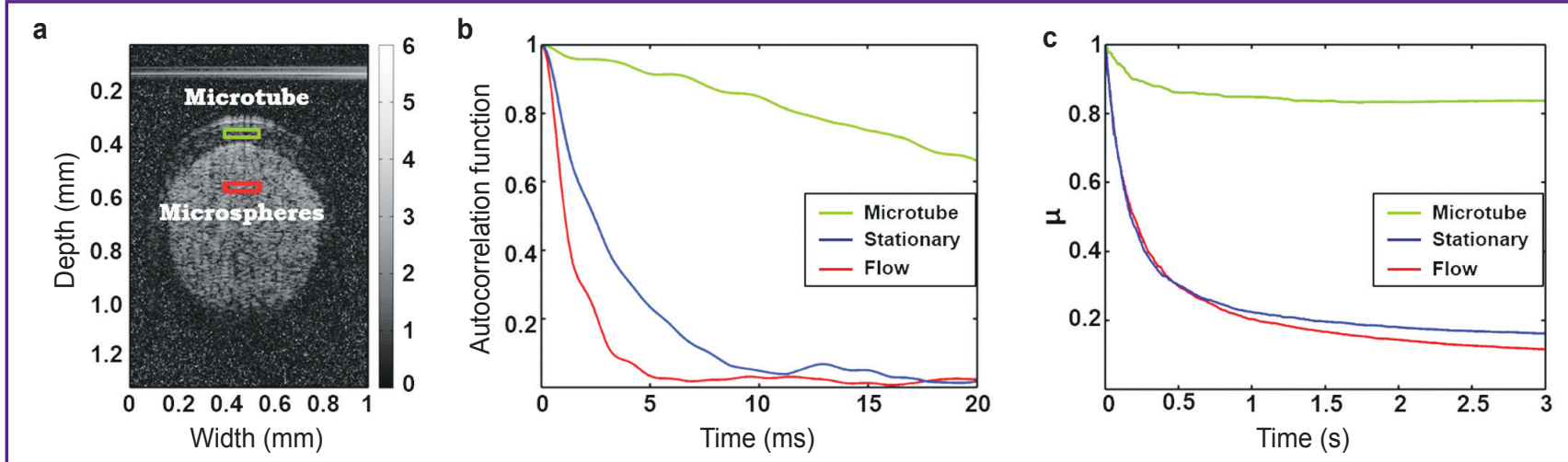

Figure 5. Water phantom with immersed $0.6-\mathrm{mm}$-diameter microtube filled with $1 \%$ polystyrene microspheres suspension: (a) OCT cross-sectional image of the phantom with microtube filled with stationary suspension; (b) averaged autocorrelation function for chosen ROls labelled in (a) with green (microtube wall) and red (polystyrene microspheres) rectangles; (c) averaged index of synchronization $\mu$ for chosen ROls

In the phantom studies, ROls were chosen as shown in Figure 5 (a) in regions within the phantom body, and within the stationary or flowing liquid. ACFs were computed for each pixel in the chosen ROls and then averaged. In order to compare ACF's decay with the new speckle synchronization metric $\mu$, index of synchronization $\mu$ was calculated for the same ROls. Decorrelation times for the chosen ROls were estimated at 1/e level.

For tissue imaging, functional speckle variance OCT [15] was used for in vivo microvasculature detection (to select one of the synchronization ROls within a big blood vessel). OCT images were taken over a $6 \times 6 \mathrm{~mm}$ field of view with $800 \mathrm{~A}$-scans per frame and a gate length of $\mathrm{N}=8$ (number of sequential same-location B-scans), to enable inter-frame comparison required for speckle variance (SV) OCT analysis. The inter-frame intensity variance from the same spatial location was calculated, with the blood vessels contrast arising from differences in time-varying speckle properties at each pixel:

$$
\mathrm{SV}_{z x}=\frac{1}{N} \sum_{t=1}^{N}\left(I_{z x t}-\bar{I}_{z x}\right)^{2}
$$

where $N$ is the number of OCT B-scans acquired at the same spatial location within a tissue volume, $I_{z x t}$ is the intensity of the $(z, x)_{\text {th }}$ pixel of the $t$-th B-scan, $z$ is the axial coordinate, $x$ is the lateral coordinate and $\bar{I}_{z x}$ is the mean intensity of $i$ pixels from $N$ consecutive B-scans. Obtained vascular map was then depth-encoded with greenyellow-red-grey-black colour map (256 colour gradations, green $=$ top tissue layers just below the glass coverslip, black $=$ deepest tissues).

\section{Results and Discussion}

We first compare ACF and index of synchronization for selected ROls in phantom with controlled optical properties based on polystyrene microspheres. The absorption of polystyrene is negligible in near infrared range [35], thus scattering is the main determinant of light transport in this phantom model. Assuming that the scattering volume is defined by the Gaussian profile of the illuminating laser beam and the system's coherence length [36], there would be a large number of identical particles within the resolution element of the system. In other words, each analysed pair of pixel intensities within the polystyrene suspension ROI (see Figure 4) 
is expected to represent the pair of averaged scattering events from many particles of same size and (spherical) shape.

Figure 5 (a) shows the OCT cross-sectional image of the phantom with microtube filled with stationary suspension. ROIs were selected within the microtube wall (green rectangle), and within the microtube lumen for the microspheres suspension (red rectangle - both stagnant and flow conditions examined). ACF analysis (Figure 5 (b)) shows that microtube wall pixels (green curve) decorrelate much slower than stationary (blue curve) and flowing (red curve) suspension with decorrelation times of the latter yielding 3.3 and $1.5 \mathrm{~ms}$ at $1 / \mathrm{e}$ level, respectively. The faster decorrelation in the presence of flow makes sense and has been previously reported by several groups [37-39]. We thus note that flow conditions do make a difference, and all ACF curves tend to zero with increasing time as expected.

Interestingly, as shown in Figure 5 (c), calculated speckle synchronization indices for the same ROls demonstrate different trends. (1) $\mu$ does not tend to zero with time, but rather asymptotes to finite levels in different phantom regions ( 0.84 level for microtube wall; 0.17 for stationary and 0.11 for flowing suspensions); (2) the time scales are much longer, compared to msrange ACFs numbers; it takes several seconds for $\mu$ to stabilize; (3) the 1/e times are now $\sim 0.5 \mathrm{~s}$ for both no-flow and flow regimes. (1) and (2) are likely the direct result of the defining calculation of $\mu$ (Eq. (5)), and as such these results are not surprising. However, the finding (3) that $\mu$ values are very similar for both no-flow and flow regimes is both novel and unexpected. We do not have a satisfactory explanation for this but posit that is has something to do with the large number of identical symmetrical scatterers being interrogated within the two speckle spots of the OCT scattering volume. Unevenly shaped scatterers like red blood cells $(\mathrm{RBC})$ in tissue will likely exhibit a larger difference in $\mu$ values for stationary versus flowing regimes.

To test this conjecture, we turn to our second phantom system, comprised of agar (to mimic biological tissue) containing an embedded microtube filled with blood. Analogous to Figure 5, Figure 6 (a) shows OCT crosssectional images of the phantom with microtube filled with stationary blood. Agar and blood ROls were chosen as indicated in (a). ACF analysis (Figure 6 (b)) shows that while agar pixels decorrelate within $\sim 160 \mathrm{~ms}$, stationary and flowing blood decorrelate much faster ( 20 and $4 \mathrm{~ms}$, respectively), which is in agreement with previously reported measurements [39]. With increasing time, ACFs tend to approach zero as in the previous experiment. Here slower signal decorrelation times for blood as well as the higher ratio of no flow/flow decorrelation times $(20 / 4=5$ for blood compared to $3.3 / 1.5=2.2$ for polystyrene microspheres) were detected. The observed decrease in decorrelation times, as pointed out above, may be due to the difference in shape; however other factors such as size and concentration of RBC are also different compared to microsphere experiments. Although symmetric microspheres can only cause scattering changes by translation [38], the more irregularly shaped RBCs have translation plus rotation as their signalchanging mechanisms, thus their decorrelation times should be faster. However, RBCs are larger than the measured microspheres, which would yield slower ACF times (both for stagnant and flow regimes). Clearly then, there are many competing factors at play, and these preliminary results and their interpretation require further research.

As in previous phantom case, in contrast to the faster decaying ACFs, the characteristic times for $\mu$ to approach its asymptotes did not change significantly. As seen from Figure 6 (c), it took nearly $3.5 \mathrm{~s}$ of data collection for agar synchronization index to stabilize at $\sim 0.66$ level
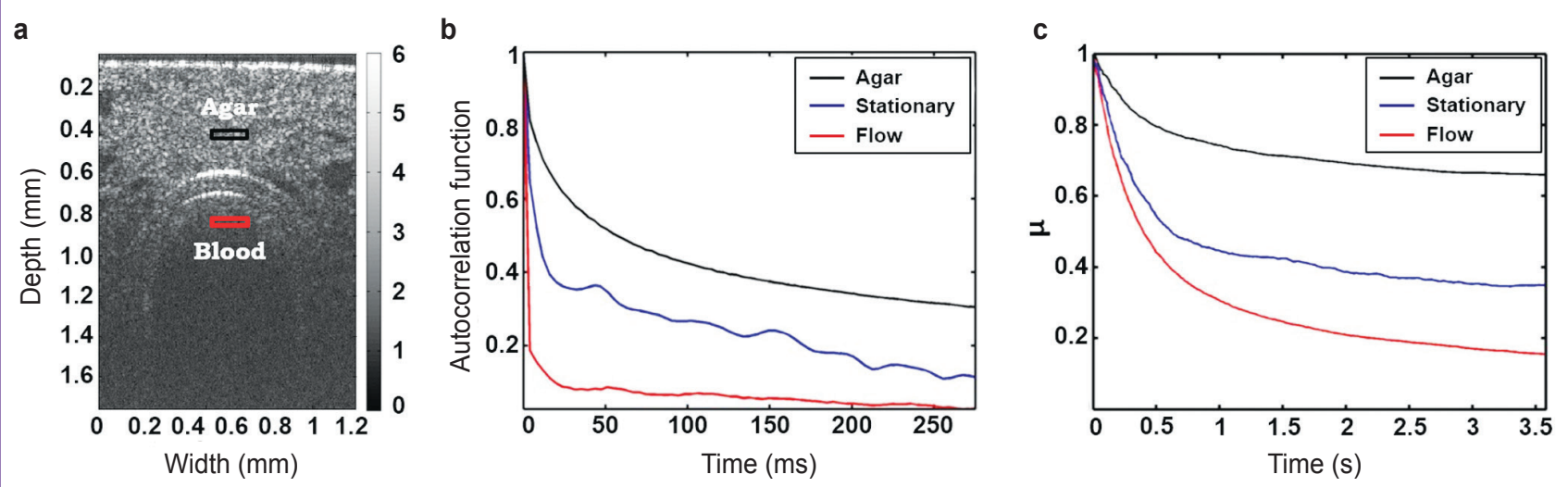

Figure 6. Agar phantom with embedded 0.6-mm-diameter microtube filled with blood:

(a) OCT cross-sectional images of the phantom with microtube filled with stationary blood; (b) averaged autocorrelation function for chosen ROls labelled in (a) with black (agar) and red (blood) rectangles; (c) averaged index of synchronization $\mu$ for chosen ROls 
(similar to microspheres suspensions), and $t \geqslant 3 \mathrm{~s}$ were also needed for flowing and stationary blood to approach "stable" values. As conjectured, $\mu$ values for irregularly shaped RBC now do exhibit a significant difference between flow and no-flow regimes $(\sim 0.15$ and 0.34 , respectively). Clearly the shape of the flowing scattering particles is important, demonstrating: (a) diffusion only (no-flow) regime with relatively slow pseudorandom changes in signal that retains synchronicity and thus yields higher $\mu$; and (b) diffusion plus flow regime with faster and less synchronized changes in signals and hence lower $\mu$. This $\mu$ sensitivity to flow and no-flow regimes may be beneficial for functional/ blocked blood vessels differentiation and is currently under consideration in our on-going in vivo studies [40]. Results for significantly longer times (up to minutes, data not shown) indicated almost no changes in $\mu$ once it stabilized at levels mentioned above. Such behaviour suggests its different nature from the single-pixel signal correlation (ACF) techniques. Indeed, this new method provides information about mutually-dependant two-pixel rhythmic changes in time, rather than time-dependent similarity in speckle intensity from a given single speckle (auto-correlation).

In the in vivo experiment, cross-sectional OCT images were taken from skin and adjacent cervical cancer tumour in a window chamber mouse model. Figure 7 (a) shows the white light photograph of the window chamber where skin, tumour, and big vessels were approximately visualized. Using OCT in speckle variance mode, we obtained the depth-encoded vasculature map and chose a slice of interest as indicated in Figure 7 (b), such as to have skin, tumour, and big vessel lumen in one cross-section. Vessel size (0.2 $\mathrm{mm}$ diameter) was chosen for its blood flow volume to be comparable with the microspheres and tissue-mimicking phantom flow volumes. Three ROls are labeled with rectangles within the OCT structural image in Figure 7 (c) (blue = skin, red= vessel lumen, black = tumour). Blood, tumour and skin decorrelation times were measured using ACF (Figure 7 (d)) to be $<1 \mathrm{~ms}, 480 \mathrm{~ms}$ and $830 \mathrm{~ms}$, respectively. These numbers reflect the fast blood flow velocity within the vessel of such a diameter [41] and higher metabolic activity of tumour cells compared to skin cells in vivo [17].

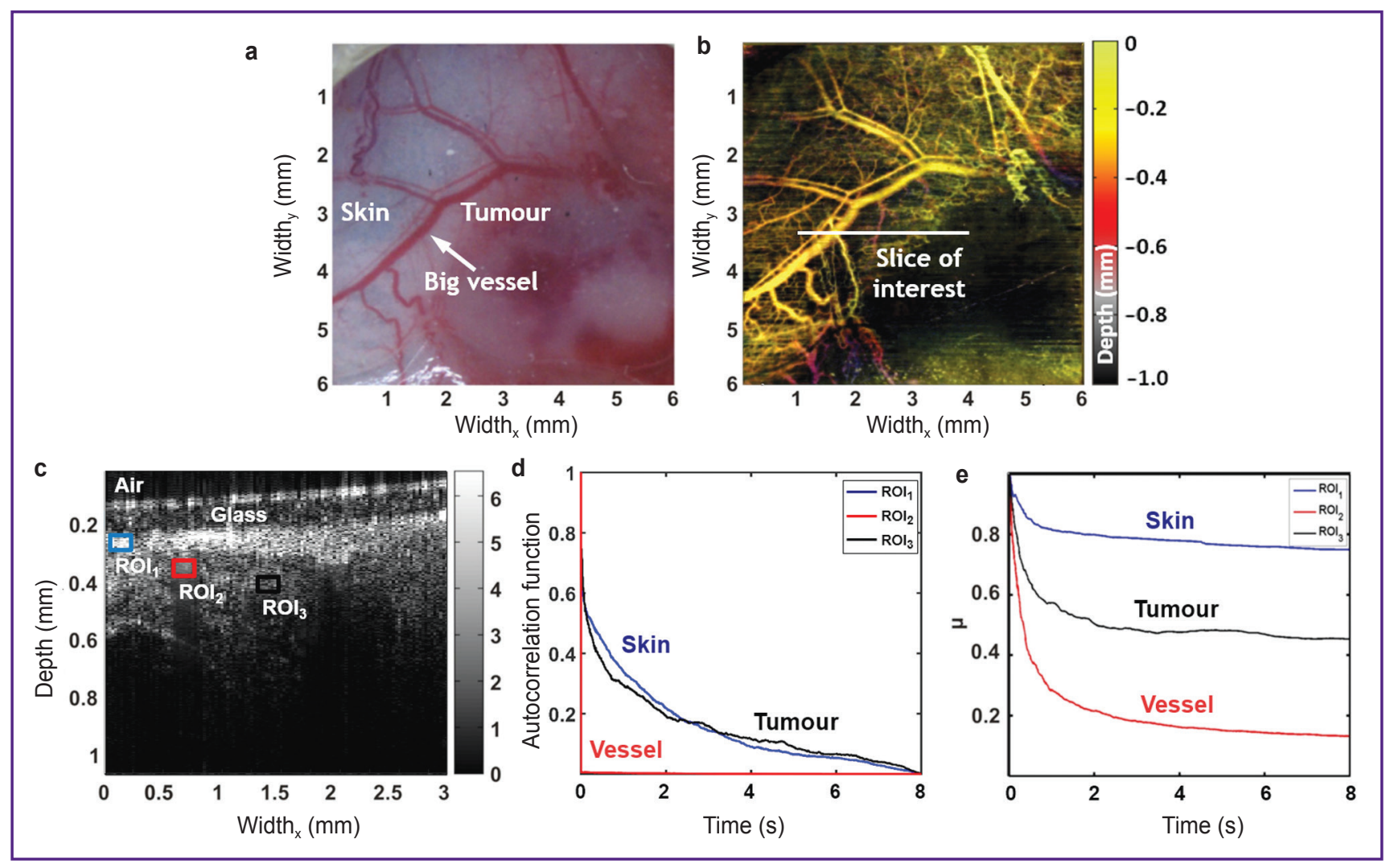

Figure 7. Initial testing of the temporal speckle synchronization method in vivo in a window chamber mouse tumour model:

(a) white light photo of the window chamber where skin, tumour, and big vessels could be differentiated; (b) depth encoded vasculature of the area shown in (a), showing the location of the selected depth slice for synchronization analysis; (c) OCT cross-sectional "slice of interest" with labelled ROls corresponding to skin ( $\mathrm{ROI}_{1}$ - blue rectangle), blood vessel $\left(\mathrm{ROI}_{2}\right.$ - red rectangle), and tumour ( $\mathrm{ROI}_{3}$ - black rectangle); (d) averaged autocorrelation function for chosen ROls; (e) averaged index of synchronization $\mu$ for chosen ROls. Asymptotic $\mu$ values were 0.74 for the skin, 0.45 for the tumour, and 0.12 for the blood at $t=8 \mathrm{~s}$ 
Similar to both phantom experiments, Figure 7 (e) shows that index of synchronization $\mu$ stabilizes with time ( $8 \mathrm{~s}$ time period is shown) to $\sim 0.74$ for skin, 0.45 for tumour, and 0.12 for blood. Comparing these in vivo tissue findings to phantom results, $\mu$ value for skin was higher than for the agar at $t=3.5 \mathrm{~s}$ (0.78 vs 0.66 ), potentially due to difference in the stiffness of the skin and incompletely solidified agar phantom. Synchronization of pixels within the blood vessel was close to $\mu$ value for the blood in the microtube at $t=3.5 \mathrm{~s}$ (0.15 vs 0.16$)$, which is reasonable considering their comparable flow volumes and similar compositions. Importantly, $\mu$ values for two tissue types (skin and tumour) were detected to be significantly different $(0.74$ vs 0.45$)$, suggesting the utility of this approach for tumour/normal tissue differentiation. Certainly comparing the results of the two types of temporal analysis - single-pixel temporal decorrelation seen in Figure 7 (d) and mutual temporal behaviour of two neighbouring pixels seen in Figure 7 (e) - we see the latter providing much greater contrast ranges for the different tissue types in vivo.

In summary, one-dimensional M-mode analysis shows the potential of the reported synchronization metric to provide a novel contrast derivable from conventional OCT structural images. In phantoms and in in vivo tissue, we provide preliminary data demonstrating its dependence and sensitivity to size/shape/movement of the correlated scatterers within the OCT interrogation volume. Some preliminary interpretations of the synchronicity trends and value levels are provided, but further work is needed to fully interpret and understand the obtained results. We are also currently implementing a two-dimensional version of the presented synchronization algorithm, to enable a parametric 2D $\mu$ image of the interrogated tissue. Challenges include maintaining a reasonable resolution while ensuring sufficient statistics within the ROI for $\mu$ estimation, optimal temporal-geometric choices for pair synchronization analysis and sufficiently long measurement time course/number of "points"/ inter-point temporal spacing to enable unambiguous $\mu$ determination. Potential solutions currently being explored include oversampling the original OCT images and faster data acquisition using OCT with $200 \mathrm{kHz}$ scanning rate or higher. Results will be reported in a separate forthcoming publication.

\section{Conclusion}

This study introduces temporal synchronization formalism to OCT imaging and presents preliminary experimental results from phantoms and in vivo tumour xenografts. A novel functional method based on speckle temporal synchronization was developed, providing novel contrast derivable from conventional OCT structural images. Speckle synchronization was compared with the more common auto-correlation analysis to demonstrate its different nature. Initial in vivo experiments showed that the reported metric is sensitive to tissue type/pathology, thus potentially providing a tool for tumour segmentation from surrounding normal tissues and its quantitative evaluation. We are currently working on further interpretation of the presented data, and implementing a $2 \mathrm{D}$ version of the presented synchronization algorithm, to generate parametric $\mu$ maps and explore the resulting contrast features. Future work will also examine the generalizability of these results in other preclinical in vivo models, and explore the optimal data acquisition parameters for fast and accurate 3D volumetric imaging suitable for synchronization analysis.

Acknowledgements. This study was supported by the Canadian Institutes of Health Research and the Ministry of Science and Education, Russian Federation (IAV). V. Demidov was supported by the Alexander Graham Bell Canada Graduate Scholarship from the Natural Sciences and Engineering Research Council of Canada. O. Demidova was supported by the Ontario Student Opportunity Grant from the Ontario Student Assistance Program.

Conflict of interest. The authors declare the absence of a conflict of interest.

\section{References}

1. Fujimoto J.G., Pitris C., Boppart S.A., Brezinski M.E. Optical coherence tomography: an emerging technology for biomedical imaging and optical biopsy. Neoplasia 2000; 2(12): 9-25, https://doi.org/10.1038/sj.neo.7900071.

2. Schmitt J.M., Xiang S.H., Yung K.M. speckle in optical coherence tomography. J Biomed Opt 1999; 4(1): 95-105, https://doi.org/10.1117/1.429925.

3. Gossage K.W., Tkaczyk T.S., Rodriguez J.J., Barton J.K. Texture analysis of optical coherence tomography images: feasibility for tissue classification. J Biomed Opt 2003; 8(3): 570-575, https://doi.org/10.1117/1.1577575.

4. Farhat G., Yang V.X.D., Czarnota G.J., Kolios M.C. Detecting cell death with optical coherence tomography and envelope statistics. J Biomed Opt 2011; 16(2): 026017, https:// doi.org/10.1117/1.3544543.

5. Farhat G., Mariampillai A., Yang V.X.D., Czarnota G.J., Kolios M.C. Detecting apoptosis using dynamic light scattering with optical coherence tomography. J Biomed Opt 2011; 16(7): 070505, https://doi.org/10.1117/1.3600770.

6. Hillman T.R., Adie S.G., Seemann V., Armstrong J.J., Jacques S.L., Sampson D.D. Correlation of static speckle with sample properties in optical coherence tomography. Opt Lett 2006; 31(2): 190-192, https://doi.org/10.1364/ ol.31.000190.

7. Van der Meer F.J., Faber D.J., Aalders M.C.G., Poot A.A., Vermes I., van Leeuwen T.G. Apoptosis- and necrosis-induced changes in light attenuation measured by optical coherence tomography. Lasers Med Sci 2010; 25(2): 259-267, https://doi.org/10.1007/s10103-009-0723-y.

8. Sugita M., Weatherbee A., Bizheva K., Popov I., Vitkin A. Analysis of scattering statistics and governing distribution functions in optical coherence tomography. Biomed Opt Express 2016; 7(7): 2551-2551, https://doi.org/10.1364/ boe.7.002551. 
9. Sugita M., Brown R.A., Popov I., Vitkin A. K-distribution three-dimensional mapping of biological tissues in optical coherence tomography. J Biophotonics 2017; e201700055, https://doi.org/10.1002/jbio.201700055.

10. Lindenmaier A.A., Conroy L., Farhat G., DaCosta R.S., Flueraru C., Vitkin I.A. Texture analysis of optical coherence tomography speckle for characterizing biological tissues in vivo. Opt Lett 2013; 38(8): 1280-1282, https://doi.org/10.1364/ ol.38.001280.

11. Gossage K.W., Smith C.M., Kanter E.M., Hariri L.P., Stone A.L., Rodriguez J.J, Williams S.K., Barton J.K. Texture analysis of speckle in optical coherence tomography images of tissue phantoms. Phys Med Biol 2006; 51(6): 1563-1575, https://doi.org/10.1088/0031-9155/51/6/014.

12. Flueraru C., Popescu D.P., Mao Y., Chang S., Sowa M.G. Added soft tissue contrast using signal attenuation and the fractal dimension for optical coherence tomography images of porcine arterial tissue. Phys Med Biol 2010; 55(8): 2317-2331, https://doi.org/10.1088/0031-9155/55/8/013.

13. Sullivan A.C., Hunt J.P., Oldenburg A.L. Fractal analysis for classification of breast carcinoma in optical coherence tomography. J Biomed Opt 2011; 16(6): 066010, https://doi. org/10.1117/1.3590746.

14. Mariampillai A., Standish B.A., Moriyama E.H., Khurana M., Munce N.R., Leung M.K., Jiang J., Cable A., Wilson B.C., Vitkin I.A., Yang V.X. Speckle variance detection of microvasculature using swept-source optical coherence tomography. Opt Lett 2008; 33(13): 1530-1532, https://doi. org/10.1364/ol.33.001530.

15. Mariampillai A., Leung M.K.K., Jarvi M., Standish B.A., Lee K., Wilson B.C., Vitkin A., Yang V.X. Optimized speckle variance OCT imaging of microvasculature. Opt Lett 2010; 35(8): 1257-1259, https://doi.org/10.1364/ol.35.001257.

16. Conroy L., DaCosta R.S., Vitkin I.A. Quantifying tissue microvasculature with speckle variance optical coherence tomography. Opt Lett 2012; 37(15): 3180-3182, https://doi. org/10.1364/ol.37.003180.

17. Farhat G., Mariampillai A., Lee K.K.C., Yang V.X.D., Czarnota G.J., Kolios M.C. Measuring intracellular motion using dynamic light scattering with optical coherence tomography in a mouse tumor model. Proc. SPIE 8230, Biomedical Applications of Light Scattering VI 2012; 823002, https://doi.org/10.1117/12.908536.

18. Huygens C. Horologium oscillatorium sive de motu pendulorum ad horologia aptato demonstrationes geometricae. Paris: F. Muguet; 1673.

19. Pikovsky A., Rosenblum M., Kurths J. Synchronization: a universal concept in non-linear sciences. Cambridge University Press; 2001; https://doi.org/10.1017/cbo9780511755743.

20. Pecora L.M., Carroll T.L. Synchronization in chaotic systems. Phys Rev Lett 1990; 64(8): 821-824, https://doi. org/10.1103/physrevlett.64.821.

21. Balanov A., Janson N., Postonov D., Sosnovtseva O. Synchronization: from simple to complex. Springer-Verlag Berlin Heidelberg; 2009.

22. Rosenblum M., Pikovsky A., Kurths J., Schäfer C., Tass P.A. Chapter 9. Phase synchronization: from theory to data analysis. In: Handbook of biological physics. Elsevier; 2001; p. 279-321, https://doi.org/10.1016/s13838121(01)80012-9.

23. Bracic M., McClintock P.V.E., Stefanovska A. Characteristic frequencies of the human blood distribution system. In: McClintock P.V.E., Broomhead D.S., Mullin T.,
Luchinskaya E.A. AIP Conference Proceedings, Stochastic and Chaotic Dynamics in the Lakes. Melville, NY; 2000; p. 146-153, https://doi.org/10.1063/1.1302378.

24. Brazhe A.R., Marsh D.J., Holstein-Rathlou N.-H., Sosnovtseva O. Synchronized renal blood flow dynamics mapped with wavelet analysis of laser speckle flowmetry data. PLoS One 2014; 9(9): e105879, https://doi.org/10.1371/ journal.pone.0105879.

25. Buzsáki G. Rhythms of the brain. New York, NY: Oxford University Press; 2006, https://doi.org/10.1093/acprof:o so/9780195301069.001.0001.

26. Anagnostopoulos C.E., Holcomb W.G., Glenn W.W.L. Pacemaker synchronization. Science 1966; 153(3744): 16361637, https://doi.org/10.1126/science.153.3744.1636.

27. Mirowski M. Standby automatic defibrillator. An approach to prevention of sudden coronary death. Arch Intern Med 1970; 126(1): 158-161, https://doi.org/10.1001/ archinte.1970.00310070160014.

28. Shabunin A.V., Demidov V.V., Astakhov V.V., Anishchenko V.S. The volume of information as a measure of the chaos synchronization. Technical Physics Letters 2001; 27(6): 476-479, https://doi.org/10.1134/1.1383830.

29. Shabunin A., Demidov V., Astakhov V., Anishchenko V. Information theoretic approach to quantify complete and phase synchronization of chaos. Phys Rev E Stat Nonlin Soft Matter Phys 2002; (5 Pt 2): 056215, https://doi.org/10.1103/ physreve.65.056215.

30. Lehrman M., Rechester A.B., White R.B. Symbolic analysis of chaotic signals and turbulent fluctuations. Phys Rev Lett 1997; 78(1): 54-57, https://doi.org/10.1103/ physrevlett.78.54.

31. Paluš $M$, Komárek V, Hrnčiř Z, Štěrbová K. Synchronization as adjustment of information rates: Detection from bivariate time series. Phys Rev E Stat Nonlin Soft Matter Phys 2001; 63(4 Pt 2): 046211, https://doi.org/10.1103/ physreve.63.046211.

32. Shannon C.E. A mathematical theory of communication. Bell System Technical Journal 1948; 27(3): 379-423.

33. Cover T.M., Thomas J.A. Elements of information theory. New York: John Wiley \& Sons, Inc.; 1991, https://doi. org/10.1002/0471200611.

34. Pearson T., Shultz L.D., Miller D., King M., Laning J., Fodor W., Cuthbert A., Burzenski L., Gott B., Lyons B., Foreman O., Rossini A.A., Greiner D.L. Non-obese diabeticrecombination activating gene-1 (NOD-Rag1 null) interleukin (IL)-2 receptor common gamma chain (IL2r gamma null) null mice: a radioresistant model for human lymphohaematopoietic engraftment. Clin Exp Immunol 2008; 154(2): 270-284, https:// doi.org/10.1111/j.1365-2249.2008.03753.x.

35. Leung M.K.K. A platform to monitor tumour cellular and vascular response to radiation therapy by optical coherence tomography and fluorescence microscopy in-vivo. MSc thesis. Medical Biophysics, University of Toronto; 2010.

36. Passos D., Hebden J.C., Pinto P.N., Guerra R. Tissue phantom for optical diagnostics based on a suspension of microspheres with a fractal size distribution. J Biomed Opt 2005; 10(6): 064036, https://doi.org/10.1117/1.2139971.

37. Weiss N., van Leeuwen T.G., Kalkman J. Simultaneous and localized measurement of diffusion and flow using optical coherence tomography. Opt Express 2015; 23(3): 3448, https:// doi.org/10.1364/oe.23.003448.

38. Popov I., Weatherbee A.S., Vitkin I.A. Dynamic light scattering arising from flowing Brownian particles: analytical 
model in optical coherence tomography conditions. J Biomed Opt 2014; 19(12): 127004, https://doi.org/10.1117/1. jbo.19.12.127004.

39. Ullah H., Ahmed E., Ikram M. Monitoring of glucose levels in mouse blood with noninvasive optical methods. Laser Physics 2014; 24(2): 025601, https://doi.org/10.1088/1054$660 \times / 24 / 2 / 025601$.

40. Demidov V., Maeda A., Sugita M., Madge V.,
Sadanand S., Flueraru C., Vitkin I.A. Preclinical longitudinal imaging of tumor microvascular radiobiological response with functional optical coherence tomography. Sci Rep 2018; 8(1): 38, https://doi.org/10.1038/s41598-017-18635-w.

41. Ullah H., Hussain F., Ikram M. Optical coherence tomography for glucose monitoring in blood. Applied Physics B 2015; 120(2): 355-366, https://doi.org/10.1007/s00340-0156144-7. 